\title{
CUIDADO DE ENFERMAGEM AS PESSOAS IDOSAS: REPERCUSSÕES DO ISOLAMENTO SOCIAL
}

Larissa Chaves Pedreira'

ORCID: 0000-0001-8939-324

Claudia Feio da Maia Lima"

ORCID: 0000-0002-4718-8683

Tânia Maria de Oliva Menezes' ORCID: 0000-0001-5819-0570

Juliana Bezerra do Amaral' ORCID: 0000-0002-7465-0183

Adriana Valéria da Silva Freitas ${ }^{\text {III }}$ ORCID:0000-0003-1831-4537

'Universidade Federal da Bahia. Salvador, Bahia, Brasil.

"Curso de Enfermagem. Santo Antônio de Jesus, Bahia, Brasil.

"'Universidade Federal do Recôncavo da Bahia.
Autor Correspondente: Larissa Chaves Pedreira

E-mail: Ichavesp@ufba.br

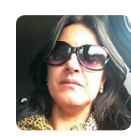

Como citar:

Pedreira LC, Lima CFM, Menezes TMO, Amaral JB, Freitas AVS. Cuidado de Enfermagem as Pessoas Idosas: Repercussões do Isolamento Social. In: Santana RF (Org.). Enfermagem gerontológica no cuidado do idoso em tempos da COVID 19. Brasilia, DF: Editora ABen; 2021. 171 p. (Serie Enfermagem e Pandemias, 5). https://doi.org/10.51234/aben.21.e05.c24

\section{INTRODUÇÃO}

Diante da condição de saúde pública que afeta o mundo e o Brasil pela COVID-19, vê-se muitas dimensões da vida dos sujeitos alteradas, estando à restrição de socialização, em destaque, acima de tudo, grupos com maior vulnerabilidade como o de pessoas idosas. $\mathrm{O}$ isolamento social em curso versa acerca de orientações da Organização Mundial da Saúde (OMS), seguidas pelo Ministério da Saúde, por meio da Agência Nacional de Vigilância Sanitária (ANVISA-MS), para redução do avanço da contaminação pelo novo coronavírus, de adoecimentos consequentes desse e mortes precoces.

$\mathrm{O}$ isolamento social foi recomendado após a pandemia do coronavírus (SARS COV 2), principalmente, para a população idosa em todo o mundo(1). Desde a chegada da pandemia ao Brasil, este segmento populacional tem sido orientado a "ficar em casa" e proteger-se de qualquer possibilidade de contaminação, evitando a doença, suas consequências e proteção do colapso do sistema de saúde, por superlotação e falta de leitos com respiradores e equipamentos adequados de proteção para a equipe de saúde.

As pessoas idosas e aquelas com comorbidades, sobretudo hipertensão e diabetes, condições prevalentes nesse grupo etário, estão mais vulneráveis às complicações da COVID-19, pelas consequências sistêmicas e respiratórias que elevam a mortalidade nessa faixa etária, diante de sua senescência e senilidade. Em estudo realizado no período de 30 dias, com 1.305 pacientes diagnosticados com a doença, a mortalidade foi maior em pessoas acima de 60 anos, com hipertensão e diabetes, e permanência de internação hospitalar entre 06 e 14 dias $^{(2)}$.

Logo, se o isolamento social de pessoas idosas as protegem da COVID-19 por um lado, por outro, pode levar a consequências também ameaçadoras e desastrosas, devendo a enfermagem atentar-se a esses riscos. $\mathrm{O}$ isolamento social antes da pandemia já era definido como uma síndrome geriátrica importante. Considerado um relevante problema de saúde 
pública, pelo perigo de agravamento/agudização de patologias pré-existentes. Assim como, o aparecimento de doenças cardíacas, autoimunes, neurocognitivas e mentais, além do elevado risco de depressão, ansiedade e suicídio(1).

Nesse contexto, o cuidado da enfermagem gerontológica se destaca por meio de estratégias de apoio, educação em saúde e monitoramento de ameaças relativas aos aspectos biológicos, sociais, espirituais e, também, concernentes à sexualidade das pessoas idosas em isolamento, evitando comprometimento a sua saúde física e mental, proporcionando o retorno saudável e seguro às atividades, quando controle da pandemia. Tais cuidados são importantes para dar maior segurança às pessoas idosas no lidar com situações adversas durante a pandemia, a exemplo da contaminação, ida aos serviços de saúde e outras.

\section{OBJETIVO}

Refletir sobre a enfermagem e seu cuidado gerontológico diante das repercussões biológicas, espirituais, sociais e relacionadas à sexualidade humana, decorrentes do isolamento social ocasionado pela pandemia do coronavírus.

\section{MÉTODOS}

Trata-se de uma reflexão teórica, a partir da leitura de textos nacionais e internacionais referentes ao tema, levantados a partir do Portal de Periódicos CAPES, acesso Café.

A busca foi orientada pela questão de investigação: Quais as repercussões do isolamento social provocado pela COVID-19 para as pessoas idosas? A partir dai, buscou-se material sobre o isolamento social em pessoas idosas, fazendo-se correlação com a situação do isolamento diante da pandemia ocasionada pelo coronavírus, por meio da leitura de textos e relatos de experiência relacionados ao tema, em sua maioria, internacionais.

Os descritores pessoa idosa, cuidados de enfermagem, isolamento social, infecções por coronavírus e pandemias foram utilizados na busca, de forma isolada ou cruzados entre si. Os artigos para leitura foram selecionados a partir de sua pertinência com a questão de estudo. A partir da leitura, reflexões foram feitas para formação de temas e discussão.

\section{RESULTADOS}

A leitura do material permitiu a formação de quatro temas para discussão: aspectos sociais; biológicos; espirituais das repercussões do isolamento para pessoas idosas; e a sexualidade humana de pessoas idosas na perspectiva do isolamento social.

\section{Aspectos sociais das repercussões do isolamento para pessoas idosas}

A inserção de pessoas idosas em grupos sociais, participando e interagindo é um dos meios de mantê-las saudáveis. Sendo assim, com a pandemia do coronavírus e a possibilidade de contrair a COVID-19, as medidas de restrição no seu no ir e vir, planejadas e executadas para mantê-las ativas, pode influenciar negativamente a qualidade de vida da pessoa idosa, com repercussões para a saúde, autonomia e independência.

Nesse sentido, vale ressaltar que, em relação aos aspectos sociais, os impactos se mostram mais evidentes, porque, atualmente, as pessoas idosas têm participado de espaços sociais através de grupos de convivência: religiosos, de família, Universidades Abertas a Terceira Idade, dentre outros. Esses grupos de convivência vêm se multiplicando desde a década de 70, acompanhando as mudanças caracterizadas pelo aumento mundial no número de pessoas idosas ${ }^{(3)}$. 
Ao serem afastadas desse convívio, quase que de maneira abrupta, pelo elevado risco de contágio que - SARS COV 2 ofereceu, a maior vulnerabilidade dessa população ao adoecimento. $E$ a solidão pode ser um aspecto que facilmente aconteça, sobremaneira, para as pessoas idosas que já vivem sozinhas em seus domicílios ou Instituições de Longa Permanência para Idosos (ILPI). Ou mesmo, aquelas que recebem familiares de maneira regular para eventos sociais (almoços de final de semana, comemorações, lazer), ou ainda, no cotidiano de famílias compostas por muitas gerações.

A solidão pode ter fatores intrínsecos como personalidade, e determinantes extrínsecos referentes à rede social de apoio percebida pela pessoa idosa. Para o autor, existem pessoas que preferem passar mais tempo sozinhas e ter uma rede social mais reduzida, definindo esse tipo de comportamento como um isolamento ativo, sem que isso implique em sentimento de solidão. Afirma, também, que podem ter pessoas mais introvertidas, que preferem baixos níveis de envolvimento social. Enfatiza que a solidão implica uma discrepância entre as preferências pessoais de envolvimento social e a rede social que o indivíduo possui, sendo compreendido como um isolamento passivo ${ }^{(4)}$.

Dessa maneira, a rede de apoio social que a pessoa idosa porventura venha possuir deve estar atenta aos sinais de solidão durante a pandemia. Esses sinais como aqueles voltados a sentimento de vazio e emoções negativas, podem levar a sintomas depressivos ${ }^{(5)}$. Para que isso aconteça, cabe reforçar que a recomendação de restrição dos contatos que as pessoas idosas passam a ter durante a pandemia da SARS COV 2, não significa a possibilidade de negligências e abandono por parte de seus familiares.

Entretanto, a falta de conhecimento e esclarecimentos do processo de envelhecimento e as alterações que esse acarreta podem levar com que a necessidade de cuidar seja realizada de forma intuitiva e equivocada, o que é um fator de risco para que os abusos e maus tratos como abandono e negligências ocorram ${ }^{(6)}$.

Diante do exposto, as consequências do isolamento social podem exacerbar o medo da doença, conduzindo as pessoas idosas para que permaneçam isoladas, sem que percebam possibilidades e estratégias capazes de minimizar os efeitos desta restrição de contato. Destarte, a enfermagem gerontológica pode orientá-las, ainda que de forma remota, para que pessoas idosas, familiares e cuidadores encontrem melhor forma de interação. Dentre algumas possibilidades, há as vídeochamadas, os e-mails ou as ligações telefônicas. É importante lembrar que as escolhas devem respeitar a autonomia e independência dessa população, para que ainda em momento da pandemia, possam se sentir e estarem ativas e valorizadas.

\section{Aspectos biológicos das repercussões do isolamento social em pessoas idosas}

O isolamento social é uma das principais medidas de proteção à pessoa idosa, visto que este grupo etário é considerado de extrema vulnerabilidade diante da pandemia do novo coronavírus.

Compreendendo a complexidade humana nos aspectos multidimensionais, as repercussões biológicas diante do isolamento social devem ser atendidas pela enfermagem como fator inicial e/ou desencadeante das demais. Portanto, a visão da enfermagem no cuidado a pessoa idosa em isolamento social deve ser multifatorial.

Nesse sentido, o distanciamento social interrompe o cotidiano de atividades diárias da pessoa idosa, como a atividade física regular. A ausência e/ou diminuição dos exercícios físicos vão interferir nos sistemas cardiovascular, respiratório e musculoesquelético favorecendo a redução da capacidade funcional e, por conseguinte, novos agravos à saúde podem surgir e a ocorrência de quedas pode ser mais frequente.

Estudo transversal ${ }^{(7)}$ com 420 idosos residentes em um estado do Brasil, em 2010, revelou que a maior parte das quedas (59\%) ocorreu no domicílio do idoso. Então, no atual momento de confinamento no ambiente domiciliar, é importante identificar as barreiras arquitetônicas que podem favorecer a queda, de acordo com a particularidade da funcionalidade de cada idoso. Ademais, a redução da massa muscular e o enrijecimento das articulações provocadas pelo possível sedentarismo entre os idosos confinados elevam esta prevalência.

Como incremento em um possível cenário favorável a quedas, destaca-se a redução da exposição ao sol, pela permanência prolongada nos domicílios, ou pela limitação do espaço físico, contribuindo para a deficiência 
de vitamina $D$ em pessoas idosas ${ }^{(8)}$. Por conseguinte, a deficiência dessa vitamina compromete o sistema ósseo, por ser responsável pela regulação de cálcio e fósforo nos ossos, como a força muscular, aumentando o risco de quedas e fraturas. Assim, o surgimento de problemas na mobilidade e no equilíbrio pode ocorrer, tornando as pessoas idosas dependentes para realização das atividades de vida diária.

O sistema imunológico também pode ser comprometido pela deficiência de vitamina $D$ e por repercussões emocionais do isolamento social. As infecções surgem com maior frequência e a susceptibilidade a internações hospitalares emergem nesse grupo etário.

Considerando a polifarmácia e a polipatologia, vale apontar que, com o isolamento social, as pessoas idosas podem ter dificuldade de acesso aos serviços e profissionais de saúde para o controle e tratamento das enfermidades. Consequentemente, a instabilidade pode expor as pessoas idosas a situações de urgência e emergência, com impactos negativos.

Então, as repercussões do isolamento social para a pessoa idosa seguirão conforme as suas reservas fisiológicas, a presença de comorbidades, a capacidade de adaptação destas pessoas a mudanças severas no seu cotidiano, a oferta de recursos tecnológicos disponíveis em um estado de pandemia, bem como a atuação gerontológica capaz de usar ferramentas digitais para atuar presencial e virtualmente na prevenção de complicações frente ao confinamento pela pandemia.

\section{Aspectos espirituais das repercussões do isolamento de pessoas idosas}

A espiritualidade é a busca pelo significado e propósito na existência, a conexão com o Eu Superior. A religiosidade é a prática da religião, podendo ser organizacional, quando o indivíduo busca o templo religioso, e não organizacional, quando é capaz de realizar sozinho, por meio da oração, leitura de textos religiosos em mensagens e livros, meditação, ouvir programas em rádio e assistir programação na televisão relativas à sua crença.

A Espiritualidade/religiosidade é uma dimensão importante na vida da pessoa idosa. Estudo refere que é um dos grupos em que a espiritualidade tem maior relevância ${ }^{(8)}$. Com isso, a enfermeira deve estar atenta para estimular a espiritualidade/religiosidade durante o isolamento social vivido na pandemia, tendo em vista que essa dimensão fica prejudicada.

O primeiro aspecto a considerar, que se apresenta comprometido, refere-se à religiosidade organizacional, quando a pessoa idosa fica impedida de se dirigir ao templo religioso. Essa ausência também se reflete nos contatos sociais suspensos de forma presencial, além de aspectos que consideram relevantes estando no templo, a exemplo de comungar e tomar passes. Outra situação é quando se formam grupos de visitas a diversas instituições, ou domicílios de pessoas da mesma religião, que passam por situações de adoecimento, hospitalização, morte de entes queridos, dificuldades econômicas, entre outras, que são práticas comuns em diversas religiões.

Apesar de estar isolado, é possível sentir medo de contrair o vírus, adoecer e morrer. Nessa condição, a espiritualidade oferece suporte, principalmente, quando pessoas de sua crença, sejam parentes ou amigos, fazem ligações e/ou chamadas de vídeo, confortando e encorajando a prosseguir confiante.

A enfermagem deve valorizar a dimensão espiritual da pessoa idosa, buscando o auxílio da família, no intuito de estimular ou estar junto nos momentos de oração e leituras, favorecendo a travessia do isolamento com menores consequências negativas para o seu bem estar e qualidade de vida.

\section{A sexualidade humana de pessoas idosas na perspectiva do isolamento social}

A pandemia de coronavírus afeta as relações humanas e, nessa perspectiva, a sexualidade está fortemente envolvida, por incluir aspectos biopsicossociais do ser humano - contatos físicos e sexuais, convivência intergeracional, relação conjugal, autocuidado e confinamento contínuo, sentimentos extremos modificadores 
do humor e'ausências' de diferentes naturezas ${ }^{10}$. É preciso salientar que, no que tange à sexualidade humana de pessoas idosas em momento tão peculiar, há duas vertentes a serem consideradas, que acabam por diferenciá-la em especificidades.

A primeira está relacionada com os aspectos afetivos, presentes ou não na vida das pessoas idosas. Para muitas, o isolamento apenas reitera uma condição pré-existente de solidão, falta de apoio e carência emocional, que giram em torno da relação de família e/ou do(a) companheiro(a). Para outros, há um sofrimento pelo afastamento, que mesmo retratando amor, por meio da proteção e do resguardo, confere difícil adaptação pela perda do convívio próximo, de afetividade pelo cuidado e do medo do adoecimento, internação e agravo à saúde.

A segunda está vinculada à saúde sexual propriamente dita, que corre com restrições mais evidentes, pela necessidade de se adotar medidas de prevenção contra o coronavírus, segundo especialistas de diferentes áreas de estudo da sexualidade humana. Mesmo sem evidências científicas acerca da sua transmissão pela via sexual, e entendendo que a sexualidade humana é um dos pilares do envelhecimento ativo e, portanto, valorizada cada dia mais por mulheres e homens mais velhos, adota-se a conduta de sua manutenção ou não, conforme a realidade de vida da pessoa idosa.

Considera-se a condição de saúde e o funcionamento social como determinantes para uma melhor opção da suspensão da prática sexual no isolamento social, em especial, daquelas com doenças crônicas prévias, sem companheiro(a) fixo(a) e convivendo em domicílios diferentes.

Por ser a transmissão do coronavírus pela via respiratória, recomenda-se a restrição de contato físico e por via oral, o que acaba por comprometer a possível desenvoltura da sexualidade ainda presente entre pessoas idosas e/ou junto a familiares, amigos e novo(a)s parceiro(a)s. Então, esse isolamento pode gerar a ideia de solidão, perda e instabilidade emocional, somado a possíveis limitações impostas pela própria idade, relativas a diferenciações ocorridas na vida sexual ao longo da vida.

Destarte, faz-se imprescindível o cuidado gerontológico de enfermagem nesse momento de adaptação a novas modalidades de se relacionar, fazendo da autoproteção um meio de ressignificação prática às pessoas idosas. É o chamado relacionamento 'blindado', no qual o maior cuidado é consigo e o desafio ser a busca por novos meios de desenvolver a sua sexualidade sem temor.

\section{CONTRIBUIÇÃO PARA ENFERMAGEM}

Esta reflexão trás como contribuição para a enfermagem a importância do monitoramento de pessoas idosas, seja atuando na atenção primária, em ILPI, ou na própria família/comunidade. É preciso que a enfermeira atente-se para um monitoramento direcionado, a partir das possíveis consequências a pessoas idosas vivenciando o isolamento social.

\section{CONSIDERAÇÕES FINAIS}

O impacto do isolamento para a população idosa quanto aos aspectos sociais, biológicos, espirituais e relacionados à sexualidade humana exigem monitoramento contínuo da enfermagem no intuito de manter a sua qualidade de vida durante a pandemia e quando controlada. Outras repercussões podem surgir diante do isolamento social e necessitam de maior atenção e aprofundamento sobre o tema e as estratégias de cuidado de enfermagem.

\section{AGRADECIMENTO}

Ao Departamento Cientifico de Enfermagem Gerontológica ABEn Nacional. 


\section{REFERÊNCIAS}

1. Armitage R, Nellums LB. COVID-19 and the consequences of isolating the elderly. Lancet Public Health. 2020[cited 2020 Jun 20];5(5):e256. Available from: https://pubmed.ncbi.nIm.nih.gov/32199471/. https://doi.org/10.1016/ S2468-2667(20)30061-X

2. Imam Z, Odish F, Gill I, O'Connor D, Armstrong J, Vanood A, et al. Am J Intern Med. 2020;10.1111/joim.13119. https://doi. org/10.1111/joim.13119

3. Schoffen LP, Santos WL. A importância dos grupos de convivência para os idosos como instrumento para manutenção da saúde. Rev Cient Sena Aires [Internet]. 2018 [cited 2018 Oct 20];7(3):160-71. Available from: http://revistafacesa.senaaires. com.br/index.php/revisa/article/\%20view/317/227

4. Rodrigues RM. Solidão, um fator de risco Rev Port Med Geral Fam 2018;34:334-8. https://doi.org/10.32385/rpmgf. v34i5.12073

5. Oliveira LMD, Abrantes GGD, Ribeiro GDS, Cunha NM, Pontes MLF, Vasconcelos SC. Solidão na senescência e sua relação com sintomas depressivos: revisão integrativa. Rev Bras Geriatr Gerontol. 2019;22(6):e190241. https://doi. org/10.1590/1981-22562019022.190241

6. Irigaray TQ, Esteves CS, Pacheco JTB, Oliveira RG, Argimon IIL. Maus tratos contra idosos em Porto Alegre, Rio grande do Sul: um estudo documental. Estud Psicol. 2016;33(3):543-51. https://doi.org/10.1590/1982-02752016000300017

7. Pelicioni PH, Lord SR. COVID19 will severely impact older people's lives, and in many more ways than you think! Braz J Phys Ther. 2020. https://doi.org/10.1016/j.bjpt.2020.04.005

8. Cruz DT, Ribeiro LC, Vieira MT, Teixeira MTB, Bastos RR, Leite ICG. Prevalência de quedas e fatores associados em idosos. Rev Saúde Pública. 2012;46(1):138-46. https://doi.org/10.1590/S0034-89102011005000087

9. Lucchetti G, Lucchetti ALG, Bassi RM, Nasri F, Nacif SAP. O idoso e sua espiritualidade: impacto sobre diferentes aspectos do envelhecimento. Rev Bras Geriatr Gerontol. 2011;14(1):159-67. https://doi.org/10.1590/S1809-98232011000100016

10. Souza JHA. Isolamento social versus qualidade de vida dos idosos: um olhar multiprofissional frente à pandemia do Covid-19. Rev Pub Saúde. 2020;3:a035. https://doi.org/10.31533/pubsaude3.a035 